\title{
Impacts of soil-soluble anions on wild and cultivated herbaceous species: implications for soil phytoremediation
}

\author{
M. J. Gutiérrez-Ginés ${ }^{1}$, A. J. Hernández ${ }^{2}$, J. Pastor ${ }^{3}$ \\ ${ }^{1}$ Department of Soil and Physical Sciences, Faculty of Agriculture and Life Sciences, PO Box 85084, Lincoln \\ University, Lincoln 7647, Christchurch, New Zealand \\ ${ }^{2}$ Department of Life Sciences, Alcalá University, 28871 Alcalá de Henares, Spain. \\ ${ }^{3}$ Department of Biogeochemistry and Microbial Ecology, National Museum of Natural Sciences, CSIC, C/ \\ Serrano 115bis, 28006,Madrid, Spain. *Corresponding author: mariajesus.gutierrez@lincoln.ac.nz
}

\begin{abstract}
This study addresses the growing problem of soil salinity. In Mediterranean regions, disused solid waste landfills have given rise to disturbed ecosystems. At such sites, both mobile soil anions and cations have toxic effects on plants. Thus, detailed knowledge of these effects is needed for the phytoremediation of soils facing the problem of salinization. In this study, high chloride levels were detected in soil samples from several sealed waste landfill sites showing metal pollution. We also examined the nutrition behaviour of six native and five cultivated herbaceous species (two forage species and three for human consumption) grown in soils containing zinc chloride. Based on the tolerance shown by Lolium rigidum to this salt in a first bioassay, we assessed its behaviour in the presence of other chlorides and other $\mathrm{Zn}$ salts (sulphates and nitrites). Besides providing plant anion concentrations (which are particulary novel results), our study identified Lolium rigidum as a species able to well-tolerate soil salinity and accumulate high levels of zinc chloride indicating its possible use for the phytostabilization of soils polluted with this salt.
\end{abstract}

Keywords: Soil and plant anions, mineral nutrition, phytotoxicity, phytoremediation

\section{Introduction}

Many ecosystems are in weak equilibrium and highly vulnerable to human interference. Human actions give rise to problems such as reduced nutrient availability with the consequence of diminished soil productivity and soil pollution. In many disturbed ecosystems, the balance of mobile anions controls the entire ion equilibrium. Anion cycles are fragile and constantly perturbed by abiotic, biotic and, above all, human factors. Hence, any study of mineral cycles should commence with exploring the proportions of soluble anions transported by water in disturbed and natural ecosystems.

Salinity has been one of the main impacts of leachates arising from uncontrolled waste landfills, 
affecting soils and adjacent water systems in both the central region of the Iberian Peninsula (Hernández et al., 1998; 1999; Pastor and Hernández, 2012) and other regions (Fatta et al., 1999; Tatsi and Zouboulis, 2002; Cheng and Chu, 2007; Kalčíková et al., 2012). Surface or subsurface leachates can affect both neighbouring soils and wet zones and water courses where such waste is usually deposited, making this the main route of pollution spread. The bioaccumulation of pollutants in the surrounding biota is another way that landfill leachates can affect the environment and its human or animal inhabitants.

Despite evidence of high salinity in this type of setting, most studies to date have focused on the impacts of heavy metals and organic compounds (Adarve et al., 1998; Gutiérrez-Ginés et al., 2014) or the effects of leachates on the growth of various plant species (Hernández et al., 1999; Cheng and Chu, 2007; Kalčíková et al., 2012) or animals (Pastor et al., 1993). Further, while numerous studies have analysed the effects of soil salinity on cultivated plants, most have centred on the effects of $\mathrm{NaCl}$, and results are presented from a physiological perspective of mineral nutrition or the biomolecular framework of the stress suffered by the plants (Cornillon and Palloix, 1997; Hall and Williams, 2003; Sharma and Dietz, 2009). Far less common have been studies centring on other chlorides or anions frequently found in soils affected by waste landfills, and anion concentrations in plant tissues have been rarely determined (Hussain and Alquwaizany, 2014; Kováčik et al., 2014).

Despite such gaps in the available literature, the topic of phytostabilization of soil covers at landfill sites will requirethe study of species that will adapt well to the climate and eco-chemical conditions of each environment. This prompted our interest in investigating the polluting actions of salts often found in landfill soils. In effect, these soils are adequate substrates for the transfer of oligonutrients to plants with possible toxic effects. As a consequence of crop irrigation mainly, soil salinization is a growing problem worldwide. There is therefore a need to improve current understanding of the impacts of salts other than sodium chloride on the plants these soils sustain.

This study was designed to identify the ions produced by surface leachates in the discharge zones of solid waste landfills that cause soil salinization and to determine the effects of different $\mathrm{Zn}$ and $\mathrm{Cl}$ salts on both grassland and cultivated plant species.

\section{Materials y Methods}

\subsection{Landfill sites and sampling}

The sites sampled for this study were 15 sealed solid waste landfills, which have been the target of on going work by our research group (Pastor and Hernández, 2010). The landfills overlie substrates representative of the central zone of mainland Spain, and their soils show an acidity-alkalinity gradient. These substrates are granite in 3 of the sites, arkoses in a further 3 , limestone in 8 and gypsum in the remaining site. Despite their different locations, the landfills have in common that they were exploited during the second half of the $20^{\text {th }}$ century in an uncontrolled manner. Waste materials (mostly mixed industrial/urban) were dumped without any form of pretreatment and the land receiving this waste had in place no impermeabilization or leachate collection system. In the 1980's and 1990's these landfills were gradually sealed. However, sealing consisted solely of applying a layer no more than $50 \mathrm{~cm}$-thick of debris and soil from the surrounding area. No further restoration measures were adopted. Most of the landfills have steep slopes (usually more than 50\%), flat areas at the top and leachate discharge areas which usually coincide with rivers, streams or even wetlands. After their capping, the landfill sites have been used for cereal cultivation, as grazing land, 
as hunting grounds or even reused for further uncontrolled waste disposal.

For the present study, sampling areas were randomly selected from the landfills' lower slopes. These zones receive surface runoff and are those mostly grazed by sheep and rabbits. The number of soil samples obtained from each landfill was proportional to its size. Factors affecting vegetation such as orientation and slope gradient were also taken into account.

Given the large presence at the sites of Gramineae (Pastor and Hernández, 2008), specimens of 3 species of this family were taken from the same collection sites as the soils to examine their element composition. These data were compared with prior data obtained in the same species growing in grasslands of the same region that lacked landfills (Pastor et al., 2012).

\subsection{Greenhouse bioassays}

As described in prior work (Pastor et al., 2014), chloride is the anion that has been mostly related to soil salinity. This anion is not a plant's basic nutrient as are nitrates, nitrites and phosphates. For this reason we chose chloride to study its possible effects on plants. Zinc was selected as the main metal detected in many landfill soil samples (Pastor et al., 2014). Zinc chloride is a soluble salt that may be easily incorporated into soil to provide different concentrations of the metal.

The species selected for the bioassays were wild species present in abundance at the sealed landfills grazed by sheep in late winter/early spring: the grasses $L o$ lium rigidum, Bromus rubens and Hordeum murinum, which grow in both acidic and basic soil covers, the leguminous species Trifolium subterraneum and Lupinus angustifolium, which grow on lower slopes with acidic soils; and Juncus buffonius, which is often abundant in early spring, especially following copious autumn rains. The early leaf stages of this species are appetizing for itinerant sheep that graze the lower landfill slopes. We also selected species that are usually sown on high flat areas and discharge areas of landfills such as wheat (Triticum aestivum, variety Anza) and barley (Hordeum vulgare, variety Volley), along with sunflower (Helianthus annus), which is often sown following cereal harvesting in this Mediterranean setting. Also included in our study were the forage leguminous species white lupin (Lupinus albus) and vetch (Vicia sativa), also often grown in this region. The seeds of wild species were collected from plants spontaneously growing at the experimental station "La Higueruela" (Toledo, Spain), which belongs to the Spanish Research Council, Consejo Superior de Investigaciones Científicas (CSIC). The seeds of cultivated species were also obtained from this station, such that they were all well-adapted to the soil and climate types.

Since acidic soils determine a greater availability of $\mathrm{Zn}$ for plants, the soil selected was an acidic soil of similar grain size and properties to the landfill soils (Pastor and Hernández, 2010) also obtained from "La Higueruela" station. Soil properties are provided in Table 1.

The soil collected was dried at room temperature and sieved through a $2 \mathrm{~mm}$ sieve. To this soil, $\mathrm{ZnCl}_{2}$ was added as one application of a solution prepared in deionized water to give final concentrations of the salt of $0,100,300,500$ or $700 \mathrm{mg} / \mathrm{kg}$. The microcosms were set up in pots containing $1 \mathrm{~kg}$ of soil as three replicates per treatment. Seeds were germinated in Petri dishes in a growth chamber and 3 seeds introduced into the pots once germinated. The bioassay was run in a greenhouse (temperature $17.4-24.5^{\circ} \mathrm{C}$ and humidity $60-70 \%$ ) for 3 months in the case of the cultivated species and for 5 months for the wild species. The pots were watered daily with $100 \mathrm{ml}$ of deionized water.

At the end of the bioassay, the plants were cut at ground level, washed in deionized water, dried in an oven at 70 ${ }^{\circ} \mathrm{C}$ for $48 \mathrm{~h}$ and their dry weights determined. 
Table 1. Chemical properties of the soil used in the bioassays

\begin{tabular}{llll}
\hline Variable & Value & Nutrient & Value \\
\hline $\mathrm{pH}$ & 5.3 & $\mathrm{Ca}(\mathrm{T})$ & 743 \\
$\mathrm{OM}$ & 1.5 & $\mathrm{Ca}(\mathrm{B})$ & 725 \\
$\mathrm{EC}$ & 86 & $\mathrm{~K}(\mathrm{~T})$ & 2282 \\
$\left.\mathrm{NO}_{2}^{-}{ }^{-} \mathrm{a}\right)$ & 1.1 & $\mathrm{~K}(\mathrm{~B})$ & 194 \\
$\mathrm{NO}_{3}{ }^{-}$ & 7.8 & $\mathrm{Mg}(\mathrm{T})$ & 1273 \\
$\mathrm{Cl}^{-}$ & 11 & $\mathrm{Mg}(\mathrm{B})$ & 109 \\
$\mathrm{~F}^{-}$ & 1.3 & $\mathrm{Fe}(\mathrm{T})$ & 5665 \\
$\mathrm{PO}_{4}{ }^{3-}$ & 14 & $\mathrm{Fe}(\mathrm{B})$ & 40 \\
$\mathrm{SO}_{4}{ }^{2-}$ & 39 & $\mathrm{Zn}(\mathrm{T})$ & 11 \\
$\mathrm{Na}(\mathrm{T})$ & 94 & $\mathrm{Zn}(\mathrm{B})$ & 2.0 \\
$\mathrm{Na}(\mathrm{B})$ & 8.6 & & \\
\hline
\end{tabular}

(a) anion concentration in $\mathrm{mg} / \mathrm{kg}$

$\mathrm{OM}=$ organic matter $(\%) ; \mathrm{EC}=$ electrical conductivity $(\mu \mathrm{S} /$ $\mathrm{cm}) ; \mathrm{T}=$ pseudototal concentration $(\mathrm{mg} / \mathrm{kg}) ; \mathrm{B}=$ exchangeable/bioavailable concentration $(\mathrm{mg} / \mathrm{kg})$.

According to the results of this bioassay in which Lolium rigidum grew well in all the treatments, in a second bioassay we subjected this species to higher $\mathrm{ZnCl}_{2}$ concentrations (900, 1100, 1300 and $1500 \mathrm{mg} / \mathrm{kg}$ ). The species was also grown in the control soil containing $700 \mathrm{mg} / \mathrm{kg}$ of other $\mathrm{Zn}$ salts $\left(\mathrm{Zn}\left(\mathrm{NO}_{3}\right)_{2}\right.$ and $\left.\mathrm{ZnSO}_{4}\right)$ and other chlorides $\left(\mathrm{CaCl}_{2}\right.$, $\mathrm{FeCl}_{3}, \mathrm{KCl}, \mathrm{MgCl}_{2}$ and $\mathrm{NaCl}$ ) to determine whether effects on growth and $\mathrm{Zn}$ and $\mathrm{Cl}$ accumulation depended on the salt. This second bioassay was run for 3 months in the same manner and under the same greenhouse conditions as for Bioassay 1 .

\subsection{Sample preparation and chemical analyses}

Dried and sieved soil samples both from the landfill sites and those used in the bioassays (control soil) were analysed to determine their chemical properties. For the control soil, we determined $\mathrm{pH}$ in slurry and organic matter by potassium dichromate reduction (Hernández and Pastor, 1989). Soil anion concentrations $\left(\mathrm{NO}_{2}^{-}, \mathrm{NO}_{3}^{-}, \mathrm{Cl}^{-}, \mathrm{F}^{-}, \mathrm{PO}_{4}{ }^{3-}\right.$ and $\mathrm{SO}_{4}^{2-}$ ) were measured by ion chromatography. The remaining elements were quantified by ICP-OES. Soil samples were subjected to extraction with a 4:1 mixture of $\mathrm{HNO}_{3}$ and $\mathrm{HClO}_{4}$ (Walsh and SSSA, 1971) to determine pseudototal metal concentrations; to extraction with $1 \mathrm{M}$ ammonium acetate at $\mathrm{pH} 7$ for exchangeable $\mathrm{Ca}, \mathrm{Mg}, \mathrm{K}$ and $\mathrm{Na}$; and to extraction with EDTA and ammonium acetate at $\mathrm{pH} 4.5$ for bioavailable concentrations of the remaining metals (Lakanen and Ervio, 1971). Dry biomass samples were ground in an IKAWERKE Yellow Line A10 grinder. Concentrations of $\mathrm{Ca}, \mathrm{K}, \mathrm{Mg}, \mathrm{Na}, \mathrm{Fe}$ and $\mathrm{Zn}$ were determined by ICP-OES following their prior extraction with a mixture of $\mathrm{HNO}_{3}$ and $\mathrm{HClO}_{4}$ (Walsh and SSSA, 1971). To measure plant anion concentrations, the method of Ghosh and Drew (1991) was modified as: $100 \mathrm{mg}$ plant material were mixed with $10 \mathrm{ml}$ of deionized water and $0.1 \mathrm{ml}$ of a $1 \%$ Tween-20 solution and left to rest for 30 minutes. The suspension was then mixed for 3 minutes in an ultrasound bath, placed on a hot plate and brought to the boil for 2 minutes. Once cool, the suspension was filtered through a nylon $0.45 \mu \mathrm{m}$ sieve and the extract made up to $25 \mathrm{ml}$ and subjected to ion chromatography on a Dionex 4500 i.

Plant specimens were also subjected to scanning electron microscopy (SEM) to visualize possible effects on leaf tissue morphology and estimate cell element contents using the method described in Gutierrez-Ginés et al. (2012). 


\subsection{Data analysis}

Plant element data were compared between the landfill and control sites using the Student's ttest. The results of the bioassays were compared between treatments by ANOVA. Data showing a non-normal distribution or not fulfilling the requirement of homoscedasticity were log transformed. Ad hoc comparisons were performed by the Bonferroni method. All statistical tests were conducted using the software package SPSS 22. In addition, nutrient ratios $(\mathrm{N} / \mathrm{P}, \mathrm{Ca} / \mathrm{P}, \mathrm{K} / \mathrm{Na}, \mathrm{Mn} / \mathrm{Fe}$ and $\mathrm{Fe} / \mathrm{Zn}$ ) were calculated for the above-ground plant samples.

\section{Results}

\subsection{Landfill soil salinity and plant nutrients}

Table 2 shows the soluble anion levels detected in the capping soils of the fifteen landfill sites. Although only landfills 14 and 15 overlie high salinity substrates, most landfills showed large differences between minimum and maximum EC values and anion concentrations. Of note, maximum EC values for landfills 3, 7 and 14 were more than 50 times higher than the minima, and $\mathrm{Cl}^{-}, \mathrm{NO}_{3}^{-}$and $\mathrm{SO}_{4}{ }^{2-}$ were the anions whose minimum and maximum concentrations varied most (300 to 7000 times higher). At some sites $\mathrm{F}^{-}, \mathrm{NO}_{2}^{-}$and $\mathrm{PO}_{4}^{3-}$ levels were also elevated. However, at all landfill sites, $\mathrm{Cl}^{-}, \mathrm{NO}_{3}{ }^{-}$and $\mathrm{SO}_{4}{ }^{2-}$ were the anions of most concern in terms of causing high salinity.

Element composition differences between specimens growing in landfills and those inhabiting grasslands were in many cases significant (Table 3 ). Despite the important problem of salinity in most of the landfills (Table 2), plants did not reflect higher concentrations of $\mathrm{Na}$ or $\mathrm{Ca}$ in their leaves. Most notable, were the greater $\mathrm{Zn}$ contents of landfill plant samples, followed by $\mathrm{Mn}$ and $\mathrm{Fe}$ concentrations. In addition, the $\mathrm{N}$ and $\mathrm{Ca}$ concentrations detected in above-ground masses of these species were higher in the grasslands.

\subsection{Effects of $\mathrm{Zn}$ chloride on wild and cultivated species}

Only two of the 11 plant species tested (B. rubens and $L$. rigidum) were unaffected by increasing $\mathrm{ZnCl}_{2}$ in the soil (Table 4). The plants H. murinum, V. sativa, T. subterraneum, J. buffonius, $H$. annus and L. angustifolius were unable to grow in the soils showing the highest $\mathrm{ZnCl}_{2}$ concentrations. Although T. aestivum, $H$. vulgare and L. albus did manage to grow at this high concentration of the salt, their growth was fairly compromised as indicated by the dry weights recorded at the end of the experiment.

Zinc and chloride levels in the above-ground biomass of the species examined were considerably higher when these were exposed to higher concentrations of these elements (Tables 5 and 6), indicating their bioavailability and easy translocation to stems and leaves. T. aestivum and $H$. vulgare showed the greater accumulation of $\mathrm{Zn}$ and $\mathrm{Cl}^{-}$, though also showed signs of toxicity. This was also observed in L. albus and even more evidently in $H$. annus, which showed above-ground levels of $3700 \mathrm{mg} / \mathrm{kg}$ of $\mathrm{Zn}$ and $41 \mathrm{~g} / \mathrm{kg}$ of $\mathrm{Cl}^{-}$when exposed to $300 \mathrm{mg} / \mathrm{kg}$ of the salt, and in L. angustifolius, showing close to $2500 \mathrm{mg} / \mathrm{kg}$ of $\mathrm{Zn}$ and $41 \mathrm{mg} / \mathrm{kg}$ of $\mathrm{Cl}^{-}$when exposed to $500 \mathrm{mg} / \mathrm{kg}$ of $\mathrm{ZnCl}_{2}$.

It should be highlighted that the species $L$. rigidum showed no signs of toxicity despite accumulating a mean of $3600 \mathrm{mg} / \mathrm{kg}$ of $\mathrm{Zn}$ and $37 \mathrm{~g} / \mathrm{kg}$ of $\mathrm{Cl}^{-}$when exposed to the maximum concentration of the salt. In contrast, $B$. rubens was the species that was least able to accumulate $\mathrm{Zn}$ and $\mathrm{Cl}^{-}$. 
Table 2. Electrical conductivity $(\mathrm{EC}, \mu \mathrm{S} / \mathrm{cm})$ and anion concentrations $(\mathrm{mg} / \mathrm{kg})$ of soil samples from 15 landfills

\begin{tabular}{|c|c|c|c|c|c|c|c|c|}
\hline Landfill & & EC & $\mathbf{F}^{-}$ & $\mathrm{Cl}^{-}$ & $\mathrm{NO}_{2}^{-}$ & $\mathrm{NO}_{3}^{-}$ & $\mathrm{PO}_{4}{ }^{3-}$ & $\mathrm{SO}_{4}{ }^{2-}$ \\
\hline \multicolumn{9}{|c|}{ Granite substrate } \\
\hline$\# 1$ & $\mathrm{M} \pm \mathrm{sd}(\mathrm{a})$ & $510 \pm 73$ & $0.3 \pm 0.0$ & $12 \pm 3.3$ & $2.1 \pm 1.2$ & $491 \pm 199$ & $9.2 \pm 4.1$ & $62 \pm 8.6$ \\
\hline $\mathrm{N}=4$ & Range & $431-606$ & $0.3-0.4$ & $7.9-15$ & $0.8-3.7$ & $363-783$ & $5.1-13$ & $53-73$ \\
\hline$\# 2$ & $\mathrm{M} \pm \mathrm{sd}$ & $525 \pm 126$ & $0.3 \pm 0.0$ & $9.1 \pm 0.5$ & $1.6 \pm 0.7$ & $365 \pm 144$ & $10 \pm 5.0$ & $33 \pm 9.7$ \\
\hline $\mathrm{N}=4$ & Range & $401-669$ & $0.3-0.4$ & $8.5-9.7$ & $1.1-2.6$ & $202-544$ & $6.2-17$ & $25-46$ \\
\hline$\# 3$ & $\mathrm{M} \pm \mathrm{sd}$ & $1158 \pm 1909$ & $1.3 \pm 2.2$ & $777 \pm 1866$ & $0.6 \pm 0.5$ & $381 \pm 556$ & $4.9 \pm 7.3$ & $219 \pm 354$ \\
\hline $\mathrm{N}=12$ & Range & $129-6690$ & $0.0-8.1$ & $19-6310$ & $0.0-1.4$ & $4.1-1848$ & $0.0-22$ & $22-1157$ \\
\hline \multicolumn{9}{|c|}{ Arkosic substrate } \\
\hline$\# 4$ & $\mathrm{M} \pm \mathrm{sd}$ & $434 \pm 522$ & $0.8 \pm 1.0$ & $26 \pm 22$ & $2.0 \pm 2.4$ & $336 \pm 702$ & $16 \pm 16$ & $49 \pm 72$ \\
\hline $\mathrm{N}=5$ & Range & $96-1346$ & $0.0-2.5$ & $5.0-59$ & $0.0-6.1$ & $0.8-1591$ & $7.3-46$ & $14-178$ \\
\hline$\# 5$ & $\mathrm{M} \pm \mathrm{sd}$ & $486 \pm 422$ & $1.0 \pm 1.7$ & $59 \pm 47$ & $1.4 \pm 3.9$ & $14 \pm 30$ & $4.4 \pm 6.9$ & $592 \pm 932$ \\
\hline $\mathrm{N}=53$ & Range & $63-2480$ & $0.0-12$ & $6.1-182$ & $0.0-28$ & $0.0-162$ & $0.0-31$ & $10-6060$ \\
\hline$\# 6$ & $\mathrm{M} \pm \mathrm{sd}$ & $173 \pm 89$ & $1.3 \pm 1.1$ & $36 \pm 36$ & $1.9 \pm 0.9$ & $37 \pm 73$ & $2.0 \pm 4.3$ & $67 \pm 46$ \\
\hline $\mathrm{N}=5$ & Range & $99-273$ & $0.4-3.1$ & $4.8-87$ & $1.3-3.0$ & $1.1-168$ & $0.0-9.8$ & $18-133$ \\
\hline \multicolumn{9}{|c|}{ Limestone substrate } \\
\hline$\# 7$ & $\mathrm{M} \pm \mathrm{sd}$ & $1061 \pm 1580$ & $1.5 \pm 1.9$ & $63 \pm 106$ & $8.9 \pm 26$ & $193 \pm 474$ & $5.1 \pm 7.4$ & $1239 \pm 1750$ \\
\hline $\mathrm{N}=50$ & Range & $145-10230$ & $0.3-13$ & $6.1-422$ & $0.0-126$ & $0.5-2674$ & $0.0-31$ & $10-7133$ \\
\hline$\# 8$ & $\mathrm{M} \pm \mathrm{sd}$ & $556 \pm 711$ & $7.2 \pm 13$ & $23 \pm 28$ & $1.2 \pm 0.9$ & $23 \pm 40$ & $0.6 \pm 1.3$ & $710 \pm 1234$ \\
\hline$N=16$ & Range & $88-2610$ & $0.4-55$ & $5.5-94$ & $0.0-3.4$ & $0.0-158$ & $0.0-4.6$ & $1.4-4549$ \\
\hline$\# 9$ & $\mathrm{M} \pm \mathrm{sd}$ & $577 \pm 348$ & $0.7 \pm 0.2$ & $74 \pm 113$ & $20 \pm 44$ & $209 \pm 217$ & $14 \pm 11$ & $229 \pm 341$ \\
\hline $\mathrm{N}=7$ & Range & $212-1210$ & $0.4-0.9$ & $6.5-319$ & $0.0-120$ & $4.9-458$ & $0.0-28$ & $14-973$ \\
\hline$\# 10$ & $\mathrm{M} \pm \mathrm{sd}$ & $534 \pm 247$ & $3.8 \pm 1.4$ & $26 \pm 25$ & $1.3 \pm 0.8$ & $48 \pm 75$ & $0.5 \pm 1.4$ & $413 \pm 283$ \\
\hline $\mathrm{N}=36$ & Range & $237-1248$ & $0.0-6.3$ & $7.7-107$ & $0.0-4.1$ & $0.9-299$ & $0.0-6.3$ & $91-1194$ \\
\hline \# 11 & $\mathrm{M} \pm \mathrm{sd}$ & $664 \pm 514$ & $0.8 \pm 0.3$ & $24 \pm 21$ & $2.6 \pm 1.5$ & $2313 \pm 6556$ & $1 \pm 1$ & $480 \pm 1015$ \\
\hline $\mathrm{N}=9$ & Range & $292-1947$ & $0.5-1.4$ & $7.6-64$ & $0.0-5.6$ & $64-19795$ & $0.0-3.2$ & $23-3164$ \\
\hline \# 12 & $\mathrm{M} \pm \mathrm{sd}$ & $283 \pm 208$ & $2.8 \pm 1.2$ & $12 \pm 7.4$ & $2.7 \pm 1.9$ & $73 \pm 106$ & $2.9 \pm 2.8$ & $93 \pm 169$ \\
\hline$N=16$ & Range & $75-759$ & $0.9-4.6$ & $4.7-31$ & $0.6-8.2$ & $0.8-409$ & $0.0-8.3$ & $1.2-677$ \\
\hline \# 13 & $\mathrm{M} \pm \mathrm{sd}$ & $188 \pm 47$ & $3.1 \pm 0.3$ & $17 \pm 11$ & $1.4 \pm 0.7$ & $52 \pm 50$ & $1.1 \pm 1.1$ & $14 \pm 3.1$ \\
\hline $\mathrm{N}=9$ & Range & $137-289$ & $2.5-3.5$ & $7.6-46$ & $0.7-2.3$ & $7.0-158$ & $0.0-3.1$ & $10-18$ \\
\hline \# 14 & $\mathrm{M} \pm \mathrm{sd}$ & $1253 \pm 2508$ & $6.2 \pm 6.4$ & $309 \pm 1281$ & $1.5 \pm 1.1$ & $74 \pm 167$ & $0.9 \pm 1.7$ & $2096 \pm 4956$ \\
\hline $\mathrm{N}=63$ & Range & $60-17290$ & $0.0-31$ & $1.1-7570$ & $0.0-7.2$ & $0.0-1152$ & $0.0-8.0$ & $8.0-27816$ \\
\hline \multicolumn{9}{|c|}{ Gypsum substrate } \\
\hline \# 15 & $\mathrm{M} \pm \mathrm{sd}$ & $2315 \pm 90$ & $0.8 \pm 0.3$ & $12 \pm 5.5$ & $1.3 \pm 0.8$ & $139 \pm 57$ & $1 \pm 1.4$ & $3958 \pm 91$ \\
\hline $\mathrm{N}=6$ & Range & $2170-2440$ & $0.5-1.3$ & $6.5-20$ & $0.6-2.7$ & $40-189$ & $0.0-3.8$ & $3791-4065$ \\
\hline
\end{tabular}

(a) $\mathrm{M}=$ mean; $\mathrm{sd}=$ standard deviation. 
Table 3. Element composition (\%) of grass species collected from landfill sites compared with values recorded in the same species collected from reference grasslands

\begin{tabular}{|c|c|c|c|c|c|c|c|c|c|c|c|c|}
\hline Species & \# samples & & $\mathrm{N}$ & $\mathrm{P}$ & $\mathrm{Ca}$ & $\mathrm{Mg}$ & $\mathrm{K}$ & $\mathrm{Na}$ & $\mathrm{Fe}(\mathrm{a})$ & $\operatorname{Mn}(a)$ & $\mathrm{Zn}(\mathrm{a})$ & $\mathrm{Cu}(\mathrm{a})$ \\
\hline \multicolumn{13}{|c|}{ Bromusrubens } \\
\hline \multirow[t]{2}{*}{ Grassland } & 4 & M & 0.99 & 0.065 & 0.32 & 0.125 & 0.45 & 0.022 & 1485 & 58 & 21 & 2.0 \\
\hline & & $s d$ & 0.20 & 0.015 & 0.08 & 0.055 & 0.15 & 0.002 & 15.0 & 12 & 1.0 & 2.0 \\
\hline \multirow[t]{3}{*}{ Landfill } & 15 & M & 1.39 & 0.11 & 0.56 & 0.24 & 0.88 & 0.02 & 865 & 79 & 90 & 1.2 \\
\hline & & $s d$ & 0.71 & 0.05 & 0.34 & 0.12 & 0.59 & 0.01 & 448 & 30 & 49 & 3.1 \\
\hline & & & & $\mathrm{F}$ & & & & & $*$ & & $* * *$ & \\
\hline \multicolumn{13}{|c|}{ Hordeum murinum } \\
\hline \multirow[t]{2}{*}{ Grassland } & 8 & M & 0.25 & 2.09 & 0.05 & 0.27 & 0.39 & 0.13 & 45 & 28 & 0.0 & 0.4 \\
\hline & & $s d$ & 0.18 & 0.82 & 0.04 & 0.22 & 0.14 & 0.04 & 21 & 12 & 0.0 & 0.5 \\
\hline \multirow[t]{3}{*}{ Landfill } & 15 & M & 1.46 & 0.27 & 0.47 & 0.20 & 0.88 & 0.05 & 585 & 66 & 70 & 0.9 \\
\hline & & $s d$ & 0.72 & 0.41 & 0.39 & 0.11 & 0.37 & 0.07 & 513 & 42 & 39 & 3.0 \\
\hline & & & $* * *$ & $* * *$ & $* *$ & & $* * *$ & $* *$ & $* *$ & $\mathrm{~F}$ & $* * *$ & \\
\hline \multicolumn{13}{|c|}{ Lolium rigidum } \\
\hline \multirow[t]{2}{*}{ Grassland } & 8 & M & 1.90 & 0.22 & 1.22 & 0.18 & 1.19 & 0.12 & 673 & 106 & 73 & 5.5 \\
\hline & & $s d$ & 0.48 & 0.04 & 0.36 & 0.06 & 0.35 & 0.11 & 688 & 72 & 39 & 2.2 \\
\hline \multirow[t]{3}{*}{ Landfill } & 14 & M & 1.46 & 0.15 & 0.47 & 0.18 & 1.33 & 0.08 & 408 & 85 & 78 & 0.8 \\
\hline & & $s d$ & 0.51 & 0.06 & 0.31 & 0.09 & 0.42 & 0.09 & 379 & 44 & 43 & 2.2 \\
\hline & & & & $\mathrm{F}$ & $* * *$ & & & & & & & $* * *$ \\
\hline
\end{tabular}

(a)concentration in $\mathrm{mg} / \mathrm{kg}$.

$* p<0.05$

$* * p<0.001$

$* * * p<0.0001$

$\mathrm{F}=$ difference at the confidence level $90 \% ; \mathrm{M}=$ mean; $\mathrm{sd}=$ standard deviation 
Table 4. Dry weights (g) per individual plant specimen (above-ground) collected at the end of the experiment

\begin{tabular}{|c|c|c|c|c|c|c|c|c|c|}
\hline \multirow{4}{*}{$\begin{array}{l}\text { Treat. } \\
\text { Control }\end{array}$} & \multicolumn{9}{|l|}{ Wild species } \\
\hline & \multirow{3}{*}{$\begin{array}{l}\text { Gramineae } \\
\text { B. rubens } \\
0.43 \pm 0.35 \text { (a) }\end{array}$} & \multirow[b]{2}{*}{ H. murinum } & \multirow{2}{*}{\multicolumn{2}{|c|}{ L. rigidum }} & \multicolumn{4}{|l|}{ Fabaceae } & \multirow{2}{*}{$\begin{array}{l}\text { Others } \\
\text { J. buffonius }\end{array}$} \\
\hline & & & & & \multicolumn{2}{|c|}{ L. angustifolium(b) } & \multicolumn{2}{|c|}{ T. subterraneum } & \\
\hline & & $2.90 \pm 0.24$ & \multicolumn{2}{|l|}{$1.8 \pm 1.4$} & $1.61 \pm 0.26$ & $\mathrm{a}$ & \multicolumn{2}{|c|}{$0.70 \pm 0.05$} & $0.22 \pm 0.16$ \\
\hline 100 & $0.30 \pm 0.06$ & $2.98 \pm 1.63$ & \multicolumn{2}{|l|}{$1.6 \pm 0.8$} & $1.16 \pm 0.27$ & $\mathrm{~b}$ & \multicolumn{2}{|c|}{$0.48 \pm 0.08$} & $0.35 \pm 0.10$ \\
\hline 300 & $0.54 \pm 0.31$ & - & \multicolumn{2}{|l|}{$2.0 \pm 1.3$} & $0.29 \pm 0.17$ & $\mathrm{c}$ & \multicolumn{2}{|l|}{0.53} & $0.28 \pm 0.12$ \\
\hline 500 & $1.12 \pm 0.68$ & - & \multicolumn{2}{|l|}{$2.1 \pm 1.6$} & \multicolumn{2}{|c|}{0.14} & \multicolumn{2}{|l|}{-} & - \\
\hline 700 & $0.49 \pm 0.54$ & - & \multicolumn{2}{|l|}{$1.7 \pm 0.5$} & & & \multicolumn{2}{|l|}{-} & - \\
\hline \multirow[t]{2}{*}{ Treat. } & \multicolumn{5}{|l|}{ Crop species } & \multicolumn{4}{|c|}{ Fodder species } \\
\hline & T. aestivum & \multicolumn{2}{|l|}{ H. vulgare } & \multicolumn{2}{|l|}{ H. annus } & \multicolumn{3}{|c|}{ L. albus } & V. sativa \\
\hline Control & $0.54 \pm 0.07$ & $1.08 \pm 1.07$ & & $3.13 \pm 0.59$ & $\mathrm{a}$ & 2.13 & 0.46 & $\mathrm{a}$ & $0.38 \pm 0.11$ \\
\hline 100 & $0.62 \pm 0.15$ & $1.15 \pm 0.91$ & & $2.24 \pm 0.77$ & a & 2.02 & 0.85 & $\mathrm{a}$ & $0.27 \pm 0.07$ \\
\hline 300 & $0.55 \pm 0.25$ & $1.06 \pm 0.39$ & & $0.23 \pm 0.20$ & $\mathrm{~b}$ & 0.67 & 0.23 & $\mathrm{~b}$ & - \\
\hline 500 & $0.33 \pm 0.07$ & $0.76 \pm 0.53$ & & - & & 0.50 & 0.34 & $\mathrm{bc}$ & - \\
\hline 700 & 0.06 & $0.19 \pm 0.15$ & - & - & & 0.16 & 0.08 & $\mathrm{c}$ & - \\
\hline
\end{tabular}

(a) Results expressed as means \pm standard deviation.

(b) Different letters indicate significant differences between treatments (Bonferroni, 95\%)

Table 5. Zinc concentrations $(\mathrm{mg} / \mathrm{kg})$ recorded in the above-ground biomass of the species tested

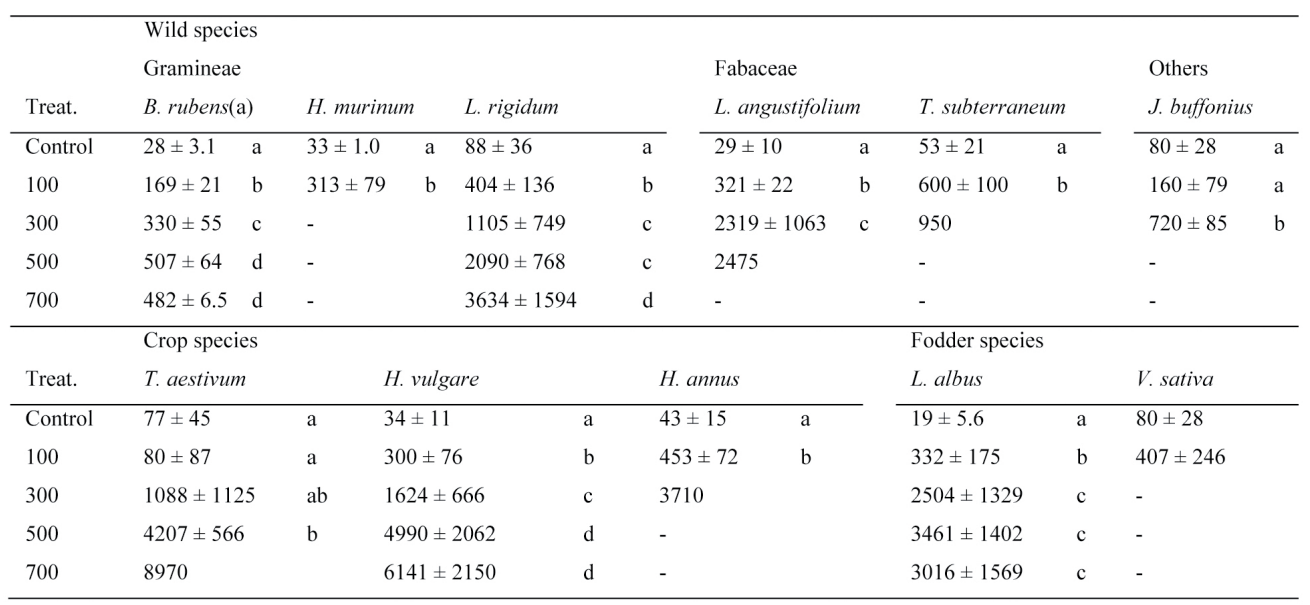

(a) Results expressed as means \pm standard deviation. Different letters indicate significant differences between treatments (Bonferroni, 95\%). 
Table 6. Chloride concentrations $(\mathrm{g} / \mathrm{kg})$ recorded for the above-ground biomass of the species tested

\begin{tabular}{|c|c|c|c|c|c|c|c|c|c|c|c|c|}
\hline \multirow{4}{*}{$\begin{array}{l}\text { Treat. } \\
\text { Control }\end{array}$} & \multicolumn{12}{|l|}{ Wild species } \\
\hline & \multicolumn{6}{|l|}{ Gramineae } & \multicolumn{4}{|l|}{ Fabaceae } & \multirow{2}{*}{\multicolumn{2}{|c|}{$\begin{array}{l}\text { Others } \\
\text { J. buffonius }\end{array}$}} \\
\hline & \multicolumn{2}{|l|}{ B. Rubens } & \multicolumn{2}{|c|}{ H. murinum } & \multicolumn{2}{|l|}{ L. rigidum } & \multicolumn{2}{|c|}{ L. angustifolium } & \multicolumn{2}{|c|}{ T. subterraneum } & & \\
\hline & $4.5 \pm 0.86$ & $\mathrm{a}$ & $2.5 \pm 0.6$ & $\mathrm{a}$ & $2.3 \pm 2.2$ & $\mathrm{a}$ & $1.4 \pm 0.24$ & $\mathrm{a}$ & $0.56 \pm 0.10$ & $\mathrm{a}$ & $3.6 \pm 2.7$ & $\mathrm{a}$ \\
\hline 100 & $16.8 \pm 1.2$ & $\mathrm{~b}$ & $13.4 \pm 1.6$ & $\mathrm{~b}$ & $12.3 \pm 3.5$ & $\mathrm{~b}$ & $20.9 \pm 3.4$ & $\mathrm{~b}$ & $11.0 \pm 1.8$ & $\mathrm{~b}$ & $2.5 \pm 0.5$ & $\mathrm{a}$ \\
\hline 300 & $20.4 \pm 0.93$ & $\mathrm{~b}$ & - & & $17.1 \pm 6.6$ & $\mathrm{~b}$ & $42.1 \pm 10.2$ & c & 32.6 & & $12.2 \pm 2.6$ & $\mathrm{~b}$ \\
\hline 500 & $17.4 \pm 5.4$ & $\mathrm{~b}$ & - & & $34.0 \pm 4.6$ & $\mathrm{c}$ & 41.3 & & - & & - & \\
\hline 700 & $26.2 \pm 3.1$ & $\mathrm{~b}$ & - & & $37.3 \pm 9.7$ & $\mathrm{c}$ & - & & - & & - & \\
\hline \multirow[t]{2}{*}{ Treat. } & \multicolumn{7}{|l|}{ Crop species } & \multicolumn{5}{|c|}{ Fodder species } \\
\hline & \multicolumn{2}{|l|}{ T. aestivum } & \multicolumn{2}{|c|}{ H. vulgare } & \multicolumn{3}{|c|}{ H. annus } & \multicolumn{2}{|c|}{ L. albus } & & \multicolumn{2}{|l|}{ V. sativa } \\
\hline Control & $3.2 \pm 1.0$ & a & $3.5 \pm$ & & $\mathrm{a}$ & $1.4 \pm 0.15$ & $\mathrm{a}$ & $2.5 \pm$ & 0.36 & $\mathrm{a}$ & $4.2 \pm 0.40$ & \\
\hline 100 & $24.6 \pm 3.6$ & $\mathrm{~b}$ & $12.9=$ & & $\mathrm{b}$ & $12.3 \pm 1.4$ & $\mathrm{~b}$ & 15.9 & \pm 5.5 & $\mathrm{~b}$ & $10.6 \pm 10.3$ & \\
\hline 300 & $37.6 \pm 11.2$ & $\mathrm{~b}$ & $45.6=$ & & $\mathrm{c}$ & 41.8 & & 47.3 & \pm 2.3 & $\mathrm{c}$ & - & \\
\hline 500 & $40.0 \pm 10.4$ & b & 70.1 & & $\mathrm{c}$ & - & & 50.2 & \pm 12.9 & $\mathrm{c}$ & - & \\
\hline 700 & 66.3 & & $67.7=$ & & $\mathrm{c}$ & - & & 35.7 & \pm 2.0 & c & - & \\
\hline
\end{tabular}

(a) Results expressed as means \pm standard deviation. Different letters indicate significant differences between treatments (Bonferroni, $95 \%$ ).

Table 7. Dry weights (DW, g) per individual specimen of L. rigidum recorded at the end of the $\mathrm{ZnCl}_{2}$ bioassay and above-ground plant anion and nutrient concentrations $(\mathrm{g} / \mathrm{kg})$

\begin{tabular}{llllllllllll}
\hline Treat. & & $\mathrm{DW}$ & $\mathrm{Cl}^{-}(\mathrm{a})$ & $\mathrm{NO}_{3}{ }^{-}$ & $\mathrm{SO}_{4}{ }^{2-}$ & $\mathrm{Ca}$ & $\mathrm{K}$ & $\mathrm{Mg}$ & $\mathrm{Na}(\mathrm{b})$ & $\mathrm{Fe}$ & $\mathrm{Zn}$ \\
\hline Control & $\mathrm{M}$ & 1.7 & $1.9 \mathrm{a}$ & 0.28 & 4.4 & $5.8 \mathrm{a}$ & 16.0 & 2.3 & 346 & 385 & $53 \mathrm{a}$ \\
& $s d$ & 0.5 & 0.65 & 0.16 & 2.8 & 1.7 & 5.9 & 0.82 & 127 & 393 & 17 \\
700 & $\mathrm{M}$ & 2.3 & $24.2 \mathrm{~b}$ & 0.30 & 1.6 & $12.1 \mathrm{ab}$ & 21.9 & 2.8 & 425 & 218 & $4987 \mathrm{ab}$ \\
& $s d$ & 0.4 & 7.7 & 0.15 & 0.75 & 4.2 & 6.3 & 1.4 & 326 & 145 & 2014 \\
900 & $\mathrm{M}$ & 3.5 & $33.8 \mathrm{~b}$ & 1.0 & 1.8 & $17.5 \mathrm{~b}$ & 15.9 & 3.8 & 651 & 236 & $12861 \mathrm{~b}$ \\
& $s d$ & 2.7 & 20.8 & 1.5 & 2.1 & 13.0 & 6.4 & 3.4 & 455 & 259 & 7503 \\
1100 & $\mathrm{M}$ & 2.0 & $42.5 \mathrm{~b}$ & 0.30 & 1.9 & $16.4 \mathrm{~b}$ & 18.2 & 2.9 & 509 & 163 & $8616 \mathrm{ab}$ \\
& $s d$ & 0.7 & 20.4 & 0.3 & 0.17 & 7.8 & 8.1 & 2.8 & 463 & 71 & 11498 \\
1300 & $\mathrm{M}$ & 2.7 & $32.1 \mathrm{~b}$ & 0.33 & 2.1 & $17.1 \mathrm{~b}$ & 15.6 & 3.2 & 512 & 171 & $7216 \mathrm{ab}$ \\
& $s d$ & 0.5 & 23.6 & 0.53 & 0.45 & 10.6 & 3.6 & 3.7 & 482 & 110 & 11530 \\
\hline \multirow{2}{*}{1500} & & 0.8 & - & - & - & - & - & - & - & - &
\end{tabular}

(a) Different letters in the same column indicate significant differences between treatments.

(b) $\mathrm{Na}, \mathrm{Fe}$ and $\mathrm{Zn}$ concentration in $\mathrm{mg} / \mathrm{kg}$

$\mathrm{M}=$ mean: $\mathrm{sd}=$ standard deviation. 
Table 8. Dry weights (DW, g) per individual specimen of L. rigidum and above-ground anion and nutrient concentrations $(\mathrm{mg} / \mathrm{kg})$ recorded at the end of the bioassay in which $700 \mathrm{mg} / \mathrm{kg}$ of different salts were added to the soil

\begin{tabular}{|c|c|c|c|c|c|c|c|c|c|c|c|}
\hline Treat. & & DW & $\mathrm{Cl}^{-}$(a) & $\mathrm{NO}_{3}{ }^{-}$ & $\mathrm{SO}_{4}{ }^{2-}$ & $\mathrm{Ca}$ & $\mathrm{K}$ & $\mathrm{Mg}$ & $\mathrm{Na}$ & $\mathrm{Fe}$ & $\mathrm{Zn}$ \\
\hline \multirow[t]{2}{*}{ Control } & $\mathrm{M}$ & 1.7 & $1862 \mathrm{a}$ & $280 \mathrm{a}$ & $4418 \mathrm{ab}$ & $5837 \mathrm{a}$ & 16041 & 2278 & $346 a$ & 385 & 53 \\
\hline & $s d$ & 0.5 & 654 & 162 & 2846 & 1664 & 5885 & 817 & 127 & 393 & 17 \\
\hline \multirow{2}{*}{$\mathrm{ZnCl}_{2}$} & M & 2.3 & $24170 \mathrm{~b}$ & $304 \mathrm{a}$ & $1629 a$ & $12141 \mathrm{ab}$ & 21941 & 2838 & $425 \mathrm{a}$ & 218 & 4987 \\
\hline & $s d$ & 0.4 & 7705 & 149 & 746 & 4157 & 6257 & 1424 & 326 & 145 & 2014 \\
\hline \multirow[t]{2}{*}{$\mathrm{CaCl}_{2}$} & M & 1.9 & $23206 \mathrm{~b}$ & $769 \mathrm{a}$ & $1645 \mathrm{a}$ & $17898 \mathrm{~b}$ & 17493 & 3967 & $883 \mathrm{ab}$ & 190 & 270 \\
\hline & $s d$ & 0.2 & 1254 & 48 & 418 & 3681 & 1828 & 771 & 22 & 26 & 66 \\
\hline \multirow[t]{2}{*}{$\mathrm{FeCl}_{3}$} & M & 2.3 & $15401 \mathrm{~b}$ & $685 \mathrm{a}$ & $1295 \mathrm{a}$ & $10553 \mathrm{ab}$ & 15727 & 2640 & $619 a$ & 482 & 84 \\
\hline & $s d$ & 0.3 & 6063 & 10 & 336 & 2590 & 2926 & 831 & 69 & 526 & 34 \\
\hline \multirow[t]{2}{*}{$\mathrm{KCl}$} & M & 2.1 & 24938 b & $543 \mathrm{a}$ & $1430 \mathrm{a}$ & $8360 \mathrm{ab}$ & 23405 & 2161 & $592 \mathrm{a}$ & 103 & 67 \\
\hline & $s d$ & 0.3 & 1621 & 26 & 364 & 1412 & 1368 & 190 & 197 & 26 & 16 \\
\hline \multirow[t]{2}{*}{$\mathrm{MgCl}_{2}$} & M & 1.6 & $11797 \mathrm{~b}$ & $689 a$ & $3337 \mathrm{ab}$ & $11103 \mathrm{ab}$ & 14922 & 2998 & $1162 a b$ & 404 & 83 \\
\hline & $s d$ & 0.1 & 8807 & 32 & 1213 & 2125 & 2812 & 1591 & 44 & 474 & 7 \\
\hline \multirow[t]{2}{*}{$\mathrm{NaCl}$} & M & 1.3 & $13328 \mathrm{~b}$ & $747 a$ & 2229 a & $10765 \mathrm{ab}$ & 12276 & 2201 & $5243 \mathrm{~b}$ & 376 & 82 \\
\hline & $s d$ & 0.3 & 4895 & 21 & 995 & 1237 & 1352 & 440 & 1899 & 156 & 7 \\
\hline \multirow[t]{2}{*}{$\mathrm{Zn}\left(\mathrm{NO}_{3}\right)_{2}$} & M & 2.5 & $2356 \mathrm{a}$ & $4157 \mathrm{~b}$ & $1236 \mathrm{a}$ & $12830 \mathrm{ab}$ & 10879 & 3040 & $961 \mathrm{ab}$ & 641 & 4060 \\
\hline & $s d$ & 0.3 & 77 & 2658 & 860 & 528 & 1430 & 173 & 57 & 376 & 490 \\
\hline \multirow[t]{2}{*}{$\mathrm{ZnSO}_{4}$} & M & 2.5 & $1185 \mathrm{a}$ & $692 \mathrm{a}$ & $8232 \mathrm{~b}$ & $6506 \mathrm{ab}$ & 13096 & 1472 & $639 a$ & 295 & 2286 \\
\hline & $s d$ & 2.2 & 439 & 29 & 3516 & 1712 & 1155 & 404 & 183 & 108 & 587 \\
\hline
\end{tabular}

(a) Different letters in the same column indicate significant differences between treatments.M = mean: $\mathrm{sd}=$ standard deviation.

Table 9. Element percentages observed in the leaf tissue of Lolium rigidum growing in soil containing 700 or $1100 \mathrm{mg} / \mathrm{kg}$ of $\mathrm{ZnCl}_{2}$

\begin{tabular}{lll}
\hline $\begin{array}{l}\text { Element } \\
(\%)\end{array}$ & $700 \mathrm{mg} / \mathrm{kg}$ & $1100 \mathrm{mg} / \mathrm{kg}$ \\
\hline $\mathrm{Mg}$ & 4.38 & - \\
$\mathrm{Cl}$ & 0.91 & 15.37 \\
$\mathrm{~K}$ & 22.99 & 30.94 \\
$\mathrm{Ca}$ & 0.73 & - \\
$\mathrm{Fe}$ & 68.61 & - \\
$\mathrm{Zn}$ & 2.37 & - \\
$\mathrm{Cu}$ & & 44.77 \\
$\mathrm{Na}$ & & 8.91 \\
\hline
\end{tabular}

Ratios between nutrients provide information on the nutritional state of a plant species. Thus, deviations from values observed in controls could indicate stress induced by the high concentrations of $\mathrm{ZnCl}_{2}$. Nutrient ratios (N/P, Ca/P, K/Na, Mn/Fe and Fe/Zn) were calculated but results are not shown since they were not conclusive. Only the $\mathrm{Fe} / \mathrm{Zn}$ ratio showed a clear decreasing trend in plants exposed to the greater $\mathrm{Zn}$ concentrations. In all species, a reduction was observed in the $\mathrm{K} /$ $\mathrm{Na}$ ratio, especially in the higher $\mathrm{ZnCl}_{2}$ treatments. This could be attributed to the notable increase produced in Na concentrations in the above-ground mass of all species, which was around double in plants exposed to higher concentrations of $\mathrm{ZnCl}_{2}$ than control plants, and even 14 times higher in the case of T. aestivum. Variations in the remaining nutrient ratios differed according to species. The ratio N/P seemed higher for the greater 
$\mathrm{ZnCl}_{2}$ concentrations in the cases of $L$. rigidum and $H$. vulgare. The $\mathrm{Ca} / \mathrm{P}$ ratio increased slightly for $B$. rubens, and this seemed more evident in L. rigidum and T. aestivum.

In Bioassay 2, L. rigidum only showed growth problems in response to the maximum soil $\mathrm{ZnCl}_{2}$ concentration tested $(1500 \mathrm{mg} / \mathrm{kg})$. No signs of toxicity were observed for the remaining treatments (Table 7). Leaf $\mathrm{Zn}$ and $\mathrm{Cl}^{-}$concentrations increased when the salt concentration was higher in the soil, but stabilized for the $900 \mathrm{mg} / \mathrm{kg} \mathrm{ZnCl}_{2}$ treatment (Table 7). Apart from $\mathrm{Zn}$ and $\mathrm{Cl}, \mathrm{Ca}$ was the only element in leaves affected by the soil $\mathrm{ZnCl}_{2}$ treatment. Thus, while the $\mathrm{Ca} / \mathrm{P}$ ratio increased with increasing $\mathrm{ZnCl}_{2}$ levels, as observed for several species in Bioassay 1, this ratio was reduced in plants grown in soils containing more than $1300 \mathrm{mg} / \mathrm{kg}$ of $\mathrm{ZnCl}_{2}$. This also occurred for $\mathrm{K} / \mathrm{Na}$ though less evidently than in the first bioassay.

\subsection{Response shown by Lolium rigidum to non zinc chloride-induced soil salinity}

The growth of $L$. rigidum was unaffected by any of the salts added to the soils at $700 \mathrm{mg} / \mathrm{kg}$ (Table 8). Despite supplementing $L$. rigidum with different salts, the concentrations of these salts recorded in the plant's above-ground mass did not always reflect this. Thus, while $\mathrm{Zn}, \mathrm{Na}$, and the anions $\mathrm{SO}_{4}{ }^{2-}, \mathrm{NO}_{3}^{-}$and $\mathrm{Cl}^{-}$showed increasing concentrations in the aboveground parts of the plants exposed to these salts, $\mathrm{Fe}$, $\mathrm{Mg}, \mathrm{K}$ and $\mathrm{Ca}$ did not show this behaviour (Table 8). Nutrient ratios also varied with respect to controls according to the salt added. The SEM results obtained are provided in Table 9.

\section{Discussion}

The problem of soil salinity in capped landfills is an important problem that needs to be addressed if we are to restore these sites. Soil is considered saline and to compromise plant growth when EC is higher than $4000 \mu \mathrm{S} / \mathrm{cm}$ (Marschner, 2012). This was confirmed in the case of 3 landfills examined here. To best of our knowledge, this is the first detailed study of the salinity and ion composition of capping soils used for landfills. Most similar studies on landfill salinity problems have focused mainly on leachates and drainage (Fatta et al., 1999; Tatsi and Zouboulis, 2002; Kalčíková et al., 2012; Hussain and Alquwaizany, 2014). As in leachates, the main anions found hereto cause soil salinity in landfills were $\mathrm{Cl}^{-}, \mathrm{SO}_{4}{ }^{2-}$ and $\mathrm{NO}_{3}^{-}$.

Gramineae clearly showed a greater abundance in the landfill sites than in the reference grassland communities (Pastor and Hernández, 2008). This is not surprising since many are frequently found in disturbed environments forming subnitrophilic communities of the order Brometalia-rubenti-tectori. The nutrient status of the three grass species sampled in the landfills was similar to that of those collected in the grasslands, indicating a tolerance of these species to this type of disturbed environment. We have already reported that species of the Gramineae family are tolerant to disturbed polluted soils, i.e. abandoned mines with high concentrations of heavy metals in soils (GutiérrezGinés et al., 2015). We thus propose that these species should be considered for remediation purposes for a wide range of disturbances.

When soil $\mathrm{ZnCl}_{2}$ concentrations exceeded $300 \mathrm{mg} /$ $\mathrm{kg}$, most species had difficulties in growing. Apart from the loss of dry weight, they showed signs of toxicity when exposed to increasing $\mathrm{ZnCl}_{2}$ concentrations (chlorosis and reduced growth). These symptoms have been also described for plants cultivated in soils containing $\mathrm{NaCl}$ (Rogers and Nobles, 1991). Salinity can affect plant growth because of modified osmotic pressure, ion toxicity, and/or nutrient imbalances (Marschner, 2012). The $\mathrm{Zn}$ and $\mathrm{Cl}$ concentrations observed in leaves, as well as changes in nutrient 
ratios indicate that any of these mechanisms could be responsible for plant growth problems at higher $\mathrm{ZnCl}_{2}$ concentrations.

In response to the $300 \mathrm{mg} / \mathrm{kg}$ soil $\mathrm{ZnCl}_{2}$ treatment, $\mathrm{Zn}$ and $\mathrm{Cl}^{-}$concentrations in plants were within the toxic range, i.e. $300 \mathrm{mg} / \mathrm{kg}$ plant dry weight for $\mathrm{Zn}$, and $3.5-30 \mathrm{~g} / \mathrm{kg}$ for $\mathrm{Cl}$ depending on the tolerance of the species (Marschner, 2012). Other authors have reported higher $\mathrm{Cl}$ concentrations in plant leaves when exposed to increasing soil $\mathrm{Cl}^{-}$concentrations (Shi et al., 2015; Pushpavalli et al., 2016), and higher cation concentrations when plants grow in high salinity soils (Bhuiyan et al., 2015).

The reduced $\mathrm{K} / \mathrm{Na}$ ratio observed in most species when $\mathrm{ZnCl}_{2}$ was added to the soil was surprising, since $\mathrm{Na}$ was not added. This nutrient imbalance may also explain the reduced growth of many of the species and has often been associated with a lower tolerance to salinity (Marschner, 2012). In effect, the correction of this imbalance is one of the main strategies used to improve the quality of saline soils (Hussain et al., 2013). L. rigidum did not show this $\mathrm{K} / \mathrm{Na}$ ratio decrease in any of the treatments.

The reader is referred to Marschner (2012) for a comprehensive review of mechanisms of plant tolerance to salinity. The two species able to tolerate $\mathrm{ZnCl}_{2}$ in our experiment (B. rubens and L. rigidum) seem to show contrasting adaptation mechanisms to salinity: an exclusion mechanism indicated by low $\mathrm{Zn}$ and $\mathrm{Cl}$ uptake by $B$. rubens (the lowest of all the species tested) and an uptake mechanism indicated by high $\mathrm{Zn}$ and $\mathrm{Cl}^{-}$concentrations observed in the leaves of $L$. rigidum.

Lolium rigidum proved tolerant to high salinity and also to the trace element $\mathrm{Zn}$, since no growth problems were noted for levels up to $1500 \mathrm{mg} / \mathrm{kg}$ of $\mathrm{ZnCl}_{2}$ in soil. Up to this soil $\mathrm{ZnCl}_{2}$ concentration, the highest leaf $\mathrm{Cl}$ concentration recorded was $42 \mathrm{~g} / \mathrm{kg}$, or slightly exceeding the highest concentration found not to cause toxicity in tolerant plants like barley, spinach, lettuce or sugar beet (Marschner, 2012). Noteworthy, $\mathrm{Zn}$ concentrations in leaves of up to $8000 \mathrm{mg} /$ $\mathrm{kg}$ were recorded, which means a bioaccumulation index from soil to above-ground mass much higher than 1. According to McGrath and Zhao (2003), this species could be considered a hyper accumulator of this metal, although further research is needed to confirm this issue.

Different salts cause different deleterious effects on plant development (Shi et al., 2015). None of the salts tested on L. rigidum hindered the growth of this species. Accordingly, L. rigidum could be an excellent candidate for remediating soils affected by salinity and trace elements in capped landfills. In future studies, the effects on this species of other types of pollution found in landfills, such as high levels of trace elements and organic compounds (Gutiérrez-Ginés et al., 2014) need to be assessed. Zn concentrations in plant dry matter higher than $300-500 \mathrm{mg} / \mathrm{kg}$ can be toxic for livestock (Chaney, 1989). When L. rigidum is exposed to this metal as $\mathrm{ZnCl}_{2}, \mathrm{Zn}\left(\mathrm{NO}_{3}\right)_{2}$ or $\mathrm{ZnSO}_{4}$, it can accumulate $\mathrm{Zn}$ in concentrations even 15 times higher than those limits. Although the outstanding Zn accumulation capacity of this species is a useful characteristic for remediation purposes, this feature is at the same time a problem for trophic networks, since many landfills are given uses such as cattle grazing or hunting (Pastor and Hernández, 2010).

\section{Conclusions}

This study examines the chloride, nitrite, nitrate, fluoride, phosphate, and sulphate concentrations of 207 samples of soils taken solid waste landfills after their sealing. Based on the high levels of chlorides observed and known high concentrations of zinc at the landfill sites, we assessed the responses of several grass species to $\mathrm{ZnCl}_{2}$ in a greenhouse bioassay. 
The three grass species tested proved more tolerant to this type of salinity than leguminous species. Among these, Lolium rigidum was able to grow in soils containing $\mathrm{ZnCl}_{2}$ concentrations of up to $1300 \mathrm{mg} / \mathrm{kg}$. The nutrition responses of the 11 species analysed, both at the physiological and cytological level in the case of L. rigidum, revealed possible mechanisms whereby the plant is able to respond to the stress provoked by soluble anions.

Our study provides quantitative data on the anion levels of metal-polluted waste landfill soils and $\mathrm{Zn}$ concentrations of plants of nutritional interest to both livestock and humans, along with plant anion levels. The latter data, besides being novel in mineral nutrition studies, could serve as indicators of salt levels in the edible portions of plants.

\section{Acknowlegments}

This work was funded by the Junta de Castilla-La Mancha, Spain (POII-2014-001-A). MJGG holds a fellowship from the Fundación Ramón Areces. The authors thank Luis Cuadra and Elena Fernández for their help with the plant anion analyses.

\section{References}

Adarve, M.J., Hernández, A.J., Gil, A., Pastor, J. 1998. B, Zn, Fe and Mn content in four grassland species exposed to landfill leachates. Journal of Environmental Quality. 27, 1286-1293.

Bhuiyan, M.S.I., Raman, A., Hodgkins, D.S., Mitchell, D., Nicol, H.I. 2015. Salt Accumulation and Physiology of Naturally Occurring Grasses in Saline Soils in Australia. Pedosphere. 25, 501-511.
Cornillon, P., Palloix, A. 1997. Influence of sodium chloride on the growth and mineral nutrition. Journal Plant Nutrition. 20, 1085-1094.

Chaney, R.L. 1989. Toxic Element Accumulation in Soils and Crops: Protecting Soil Fertility and Agricultural Food-Chains. In: B. Bar-Yosef, N.J. Barrow, J. Goldshmid (eds). Inorganic Contaminants in the Vadose Zone. Springer-Verlag, Berlin, pp: $140-158$.

Cheng, C.Y., Chu, L.M. 2007. Phytotoxicity data safeguard the performance of the recipient plants in leachate irrigation. Environmental Pollution. $145,195-202$.

Fatta, D., Papadopoulos, A., Loizidou, M.A. 1999. Study of the landfill leachate and its impact on the groundwater quality of the greater area. Environmental Geochemistry and Health. 21, 175-190.

Ghosh, G., Drew, M.C. 1991. Comparison of analytical methods for extraction of chloride from plant tissue using $36 \mathrm{Cl}$ as tracer. Plant and Soil. 136, 265-268.

Gutiérrez-Ginés, M.J., Pastor, J., Hernández, A.J. 2012. Integrated approach to assessing the effects of soils polluted with heavy metals on a plant population. Ecotoxicology. 21, 1965-1978.

Gutiérrez-Ginés, M.J., Hernández, A.J., Pérez-Leblic, M.I., Pastor, J., Vangronsveld, J. 2014. Phytoremediation of soils co-contaminated by organic compounds and heavy metals: bioassays with $\mathrm{Lu}$ pinus luteus $\mathrm{L}$. and associated endophytic bacteria. Journal of Environmental Management. 143, 197-207.

Gutiérrez-Ginés, M.J., Pastor, J., Hernández, A.J. 2015. Heavy Metals in Native Mediterranean Grassland Species Growing at Abandoned Mine Sites: Ecotoxicological Assessment and Phytoremediation of Polluted Soils. In: Heavy Metal Contamination of Soils. Soil Biology, Volume 44, Springer. pp 159-178. 
Hall, J.L., Williams, L.E. 2003. Transition metal transporters in plants. Journal of Experimental Botany. 54, 2601-2613.

Hernández, A.J., Pastor, J. 1989. Técnicas analíticas para el estudio de las interacciones suelo-planta. Henares Revista de Geología. 3, 67 - 102.

Hernández, A.J., Adarve, M.J., Pastor, J. 1998. Some impacts of urban waste landfills on Mediterranean soils. Land Degradation \& Development. 9, 21-33.

Hernández, A.J., Adarve, M.J., Gil, A., Pastor, J. 1999. Soil salination from landfill leachates: effects on the macronutrient content and plant growth of four grassland species. Chemosphere. 38, 1693-1711.

Hussain, G., Alquwaizany, A.S. 2014. Ecological study of Wadi Thulaim in Al-Kharj, Saudi Arabia. Trends in Applied Sciences Research. 9, 79-92.

Hussain, Z., Khattak, R.A., Irshad, M., Eneji, A.E. 2013. Ameliorative effect of potassium sulphate on the growth and chemical composition of wheat (Triticum aestivum L.) in salt-affected soils. Journal of Soil Science and Plant Nutrition. 13, 401-415.

Kalčíková, G., Zagorc-Končan, J., Zupančič, M., Žgajnar, A. 2012. Variation of landfill leachate phytotoxicity due to landfill ageing. Journal of Chemical Technology and Biotechnology. 87, 1349-1353.

Kováčik, J., Štěrbová, D., Babula, P., Švec, P., Hedbavny, J. 2014. Toxicity of Naturally-Contaminated Manganese Soil to Selected Crops. Journal of Agriculture and Food Chemistry. 62, 7287-7296.

Lakanen, E., Ervio, R. 1971. A comparison of eight extractants for the determination of plant available micronutrients in soils. Acta Agralia Fennica. 123, 223-232.

Marschner, P. 2012. Marschner's Mineral Nutrition of Higher Plants. $3^{\text {th }}$ Edition. Elsevier, London, 651 p.
McGrath, S.P., Zhao, F.J. 2003. Phytoextraction of metals and metalloids from contaminated soils. Current Opinion in Biotechnology. 14, 277-282.

Pastor, J., Hernández, A.J. 2008. Multi-functional role of grassland systems in the ecological restoration of mines, landfills, roadside slopes and agroecosystems. Options Mediterranéennes. 79, 103-107.

Pastor, J., Hernández, A.J. 2010. Descripción de vertederos sellados de la Comunidad de Madrid. In: A.J. Hernández, C. Bartolomé (eds). Estudio multidisciplinar de vertederos sellados. Caracterización y pautas de recuperación. Servicio Publicaciones Universidad de Alcalá, Alcalá de Henares, Spain, pp: 13-33.

Pastor, J., Hernández, A.J. 2012. Heavy metals, salts and organic residues in old solid urban waste landfills and surface waters in their discharge areas: Determinants for restoring their impact. Journal of Environmental Management. 95, S42S49.

Pastor, J., Alía, M., Hernández, A.J., Adarve, M.J., Urcelay, A., Antón, F.A. 1993. Ecotoxicological studies on effects of landfill leachates on plants and animals in Central Spain. The Science of the Total Environment. 140, 127-134.

Pastor, J., Gutiérrez-Ginés, M.J., Hernández, A.J. 2012. Composición nutricional de especies de vertederos sellados con suelos ácidos pastados por ovinos. In: I. Bonilla, P. Zornoza, A. Gárate, E. Marco, R. Rivilla, J.J. Lucena (eds). La nutrición mineral de las plantas como base de una agricultura sostenible. XIV Simposio HispanoLuso de Nutrición Mineral de las Plantas. Nutriplanta. 2012, pp: 357-363.

Pastor, J., Gutiérrez-Ginés, M.J., Bartolomé, C., Hernández, A.J. 2014. The complex nature of pollution in the capping soils of closed landfills: case study in a Mediterranean setting. In: M.C. 
Hernández-Soriano (ed). Environmental Risk Assessment of Soil Contamination. InTech, Leuven, Belgium, pp: 199-223.

Pushpavalli, R., Quealy, J., Colmer, T.D., Turner, N.C., Siddique, K.H.M., Rao, M.V., Vadez, V. 2016. Salt Stress Delayed Flowering and Reduced Reproductive Success of Chickpea (Cicer arietinum L.), A Response Associated with $\mathrm{Na}^{+} \mathrm{Ac}-$ cumulation in Leaves. Journal of Agronomy and Crop Science. 202, 125-138.

Rogers, M.E., Nobles, C.L. 1991. The effect of $\mathrm{NaCl}$ on the establishment and growth of balance clover (Trifolium micheliaum savi var Balasae Buiss). Australian Journal of Agricultural Research. 42, 847-857.
Shi, L., Ma, S., Fang, Y., Xu, J. 2015. Crucial variations in growth and ion homeostasis of Glycine gracilis seedlings under two types of salt stresses. Journal of Soil Science and Plant Nutrition. 15, 1007-1023.

Tatsi, A.A., Zouboulis, A.I. 2002. A field investigation of the quantity and quality of leachate from a municipal solid waste landfill in a Mediterranean climate (Thessaloniki, Greece). Advances Environmental Research. 6, 207-219.

Walsh, L.M., Soil Science Society of America. 1971. Instrumental Methods for analysis of soils and plant tissue, vol VII. Soil Science Society of America, Wisconsin. 